\title{
An Enhanced loT Based Tracing and Tracking Model for COVID -19 Cases
}

\author{
Sakthi Jaya Sundar Rajasekar ${ }^{1}$ (D)
}

Received: 9 November 2020 / Accepted: 17 November 2020 / Published online: 18 January 2021

๑) Springer Nature Singapore Pte Ltd 2021

\begin{abstract}
The COVID-19 pandemic has alarmed the world nations to impose strict curfews and emergencies to prevent the social transmission of the disease. In order to achieve this, effective Tracing and Tracking of the suspected COVID-19 cases need to be achieved. In view of the enormous number of cases being recorded each day, this process couldn't be performed effectively with simple manual tracing. Hence, we have proposed an Internet of Things (IoT) based automated Tracing and Tracking method for identification of the possible contacts with deployment of cost-effective RFID Tags and the mobile of the individuals which act as a reader. Thereby, tracing of persons who have crossed the subject would be possible even without the knowledge of the suspected cases. This would enable cent percent quarantine of possible primary and secondary contacts and monitoring of the same by the administrative agencies. This would augment the nations' capability of managing the pandemic.
\end{abstract}

Keywords COVID-19 $\cdot$ Internet of Things (IoT) $\cdot$ Near Field Communication (NFC) $\cdot$ Radio Frequency Identification (RFID) tag

\section{Introduction}

A highly contagious pneumonia outbreak took place in the month of December 2019 in the Chinese city of Wuhan. This outbreak was caused by the Novel Coronavirus named SARS-CoV-2. This disease is designated as COrona Virus Disease-19, abbreviated as COVID-19. This disease was announced as a pandemic by the World Health Organization (WHO). The disease has travelled to several countries and has infected nearly 50 million people around the globe, leaving more than 1.2 million dead [1]. Although various drugs are in various stages of trial all around the globe, none of them has yet been declared as the standard drug in the treatment protocol. Vaccines for COVID-19 are yet to be developed and with lack of sufficient diagnostic kits,

This article is part of the topical collection "Advances in Computational Approaches for Artificial Intelligence, Image Processing, IoT and Cloud Applications" guest edited by Bhanu Prakash K N and M. Shivakumar.

Sakthi Jaya Sundar Rajasekar

drsakthi03@gmail.com

1 Melmaruvathur Adhiparasakthi Institute of Medical Sciences and Research, Melmaruvathur, Chengalpattu District, Tamil Nadu 603319, India several countries have been crumbling under the pressure of the health care emergency and economic recession as majority of the affected nations have enforced a partial or complete lockdown. The countries are no longer able to bear the impact of the pandemic on the economy and hence, are reopening. This is a phase where countries should exercise utmost caution where aggressive testing could be the only way forward. We need to test, isolate and treat patients. The main issue nations face right now is tracing people who have had contact with COVID-19 suspected or positive cases. When the cases were very less, manual tracing has proved successful. However, with each day recording very high numbers, we need to look at a more automated, efficient and a cost-effective tracing protocol which should be easily usable and acceptable by the masses. The proposed work aims to resolve this issue using the Internet of Things (IoT) Technology with an efficient and cost-effective model. This in turn helps to prevent the social transmission of the disease by breaking the chain of transmission. This could eradicate the disease at the earliest possible.

The Radio Frequency Identification (RFID) Tag is one of the sensor devices in the edge layer of the Internet of Things (IoT) technology, which is used to uniquely identify a thing (or person) in the world and make an entry in a registry. This 
technology is being used in various fields from agriculture to industries.

\section{Related Works}

These RFID tags have a wide range of applications in various fields which include asset tracking within hospitals. Simulation of managing the emergency department with RFID tag is discussed [2]. Survey about the usage of RFID tag for managing the safety of the patients is presented [3]. Survey on Physical Activity monitoring using wearable is briefed [4]. Near Field Communication (NFC) based health care management system for managing the patients' details in the hospital server for further processing and helping the concerned doctors to make better informed decisions is discussed [5, 6]. Development of Healthcare monitoring system using machine learning algorithms, in a remote setting is discussed [7]. The various prospects and barriers of using the RFID Technology is discussed. Usage of RFID Technology in Medical equipment tracking, medical personnel tracking, tracking of drug consignments within the hospital is discussed [8]. An overview of prospects of IoT in Medical environment and the challenges in its global acceptance is discussed [9]. Application of IoT Technology in Electronic Health Monitoring proves to be a game changer. Effective diagnosis, regular monitoring, storing vital medical data using IoT is discussed [10]. Temperature and heartbeat sensors are being integrated through IoT to effectively monitor old aged peoples' health to inform their loved ones about any abnormality and adverse events like heart attacks are discussed in [11]. IoT Technology is proving to be fruitful in providing healthcare services even in extremely remote areas. Vitals like Temperature and Pulse rate are being used to monitor the health status of an individual [12]. IoT, when augmented with Machine Learning Techniques have been able to detect coronary illnesses much earlier. This could be employed in a mass screening for presence of coronary illnesses in remote areas [13]. Aggregation of various bodily parameters using Raspberry Pi to predict chronic disorders is discussed [14]. Various body vital signs are recorded in real time and used to analyze the health status of an individual, thereby eliminating the need for frequent hospital visits.

Architectural review of the smart IoT-powered Healthcare system is discussed [15]. During this COVID-19 pandemic, models like these would be really beneficial. Various contact tracing apps and their nationwide implementation is discussed. The application's attributes and user's concerns regarding security and privacy are briefed [16]. Usage of Wearables in contact tracing and an overview of the existing solutions are discussed [17]. Performance evaluation of various techniques like Wi-Fi, Bluetooth and GPS in contact tracing is discussed. A novel model used to evaluate the price and efficacy of the various contact tracing technologies of COVID-19 are briefed [18]. A stochastic transmission model is used to study the efficacy of the local responses undertaken to battle the pandemic. It is used to analyze the usefulness of contact tracing and isolating suspected cases in management of the pandemic [19].

\section{Materials and Methods}

This paper aims at developing an IoT based Tracing and Tracking model for COVID-19 Cases using Radio Frequency Identification Technology. The architecture of the proposed model is shown in Fig. 1. The proposed model works with a High Frequency Passive RFID Tags. The High Frequency RFID Tags have a range of 1 meter and operates over a frequency of $13.56 \mathrm{Mega}$ Hertz (MHz). These tags are classified as active and passive depending on the availability of power source. The active RFID possess its own power source. The passive RFID Tags doesn't have a power source. The tag gets energized from the Electromagnetic Induction from the reader [20]. In our model, the Near Field Communication (NFC) protocol present in the mobiles enable mobile phones to act as a reader. The High Frequency passive RFID Tags are provided to the citizens by local administrative agency, which consists of various details like their name, age, sex, communication details, photograph, history of smoking, COVID-19 related details like fever, cold, cough, history of travelling, comorbid factors (if present) such as diabetes, hypertension, Chronic Kidney Disease(CKD), cancer, previous surgeries, any organ transplants and history of intake of immunosuppressive drugs as represented in Fig. 2.

A mobile app is developed and installed in the mobile phone which keeps running in the background. If and when a person, who is tagged with a High Frequency RFID Tag crosses a mobile phone it gets recorded and the collected details from the mobile app are passed to the edge device for further processing. The tracking expert system would be running in edge system. This record is more fool-proof than human tracing as this even records people who goes beyond the person's visibility (line of sight) and remembrance. The details collected through the NFC (which acts as RFID reader) present in a mobile of the suspected case is passed on to the edge system for further processing that should be reviewed by the local administrative agency who distribute the RFID tags. Later if anyone is identified as a suspected case on development of symptoms, the persons whom he/ she has crossed would be identified from the edge device. Subsequently, they are alerted through their mobile app present in their mobiles, thereby alarming them of a possible infection and to make their quarantine stricter and approach the nearest health care facility even on development of mild 


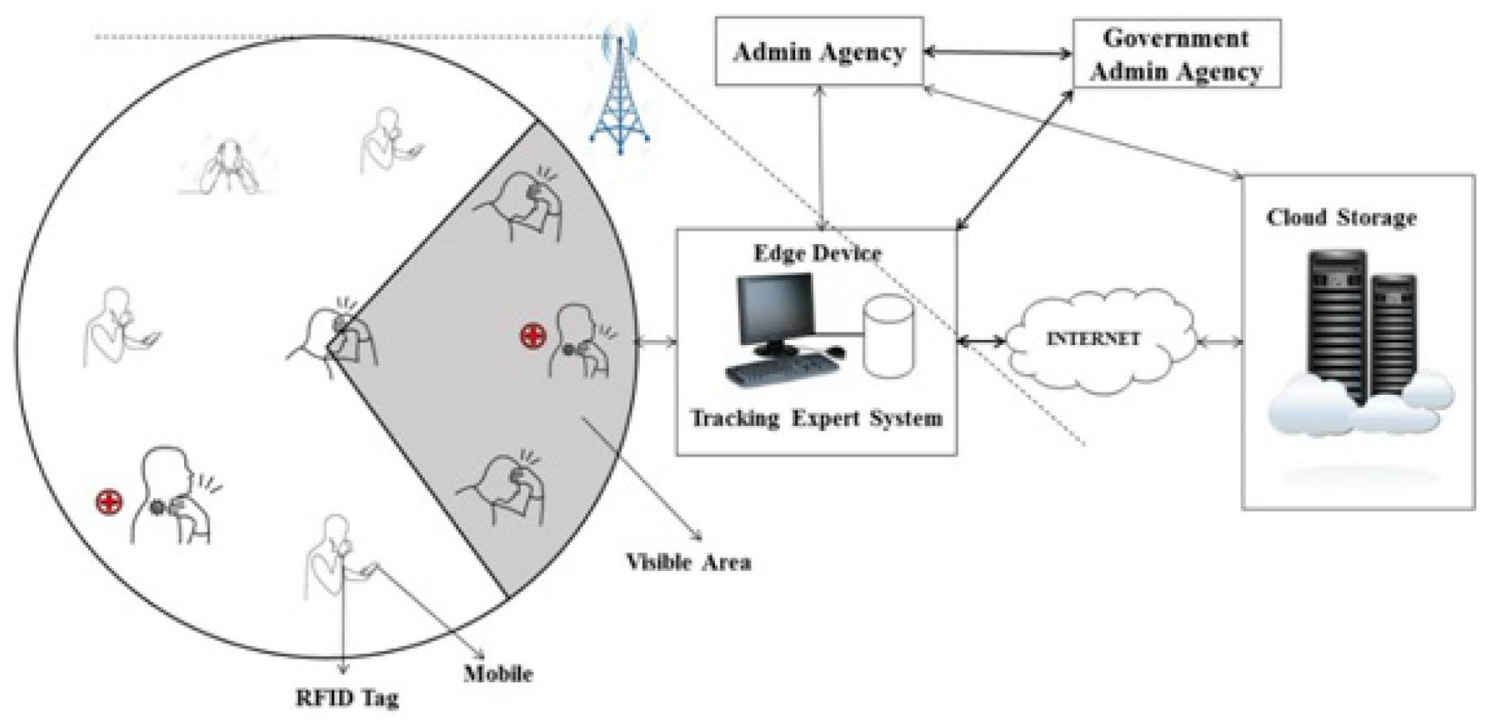

Fig. 1 Architecture of the proposed model

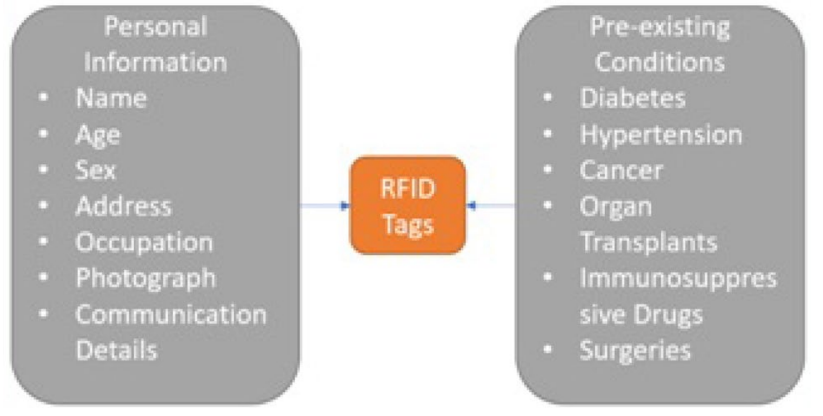

Fig. 2 Data stored in RFID Tags

symptoms. However if the suspected subject is found to be COVID-19 positive, the details of persons whom they have crossed, will be notified to the Government administrative officials and they will be placed under strict quarantine. Also the risk of these persons acquiring the infection will be assessed based upon the scoring pattern, which is based on the parameters like age, sex, comorbid factors and other factors. All these will be used to determine the intensiveness of the isolation and effectively make a clinical decision. Also, these epidemiological data will be used to chart out a containment plan to prevent community spread of the disease.

In order to effectively battle the emergent pandemic conditions, processing of the collected details from the suspected cases through their RFID tags and mobiles will be processed by the edge devices. The backup of the processed details would be maintained in cloud to provide high reliability. Cloud computing technology is used to provide highly reliable, high end storage and processing capabilities to the end users. The required details from the tracking expert system are forwarded to the administrative authorities, nearby health care workers, the suspected cases and others those who have crossed the suspected ones to enable further actions.

\section{Results and Discussion}

The main contributions of the proposed work is to efficiently and cost effectively trace and track the persons who have been in proximity to the suspected cases to further take the necessary measures such as undergoing quarantine and treatments. A comparison between manual contact tracing and IoT-enabled Contact Tracing are given in Table 1. The proposed model effectively transmits the details to the administrative authorities to take further actions in preventing the social transmission of the disease even in case of ignorance of the suspected ones. This could help the nation to flatten the curve at the earliest and get rid of the invisible enemy, the novel coronavirus. 
Table 1 Comparison of manual contact tracing and IoT-enabled contact tracing

\begin{tabular}{lll}
\hline Parameters & Manual contact tracing & IoT-enabled contact tracing \\
\hline Efficacy & Low & High \\
Cost & High & Low \\
Health Care Workers (HCWs) & More HCWs are needed & Less HCWs are needed \\
Chances of miss-outs & Significant & Nil \\
Real time surveillance & Not Possible & Possible \\
Challenges & Impossible to trace contacts of & Non availability of Smartphones \\
& enormous COVID-19 patients & \\
\hline
\end{tabular}

Author Contributions The sole author has contributed towards the preparation of the manuscript.

Funding This study did not receive any formal funding.

\section{Compliance with Ethical Standards}

Conflict of interest The author declares that he has no conflict of interest.

Ethical Approval This study doesn't involve human participants and hence, ethical approval is not required.

Consent to Participate This study doesn't involve any human participants and hence, doesn't require consent to participate.

Consent to Publish This study doesn't involve any human participants and hence, doesn't require consent to publish.

Date or Code Availability The data and code would be made publicly available upon publication.

\section{References}

1. https://covid19.who.int/. Accessed on 7th Nov 2020

2. Deryahanoglu O, Kocaoglu B. Applications of RFID systems in healthcare management: a simulation for emergency Department. Int J Innovat Technol Explor Eng (IJITEE). 2019;8(10):787-92 ISSN: 2278-3075.

3. Haddara M, Staaby A. RFID Applications and adoptions in healthcare: a review on patient safety. Int Conf Enterprice Inf Syst Proc Comput Sci 2018;138:80-8.

4. Wu M, Luo J. Wearable Technology applications in healthcare: a literature review. Online J Nurs Inf (OJNI). http://www.himss.org/ ojni

5. Bandarkavte A, Jagadeesh SD. NFC based secure healthcare monitoring system. Int J Innovat Res Comput Commun Eng 2016;4(Issue 4), 7316-7320. Available from: http://ncdc.gov.in.

6. Madhura PM, Jain P, Shankar H. NFC-based secure mobile healthcare system. Int J Adv Netw Appl (IJANA), pp. 187-190.

7. Poorani M, Vaidehi V, Varalakshmi P. Personalized Remote Health Monitoring Scheme (PRHMS) using Machine Learning and Genetic Algorithm, World Summit on Advances in Science, Engineering and Technology Cambridge Summit-2018 on January 4-6, 2018.

8. Abugabah A, Nizamuddin N, Abuqabbeh A. A review of challenges and barriers implementing RFID technology in the Healthcare sector. Procedia Comput Sci. 2020;170:1003-10. https://doi. org/10.1016/j.procs.2020.03.094.
9. Nogueira V, Carnaz G. An Overview of IoT and Healthcare (2019).

10. Singh D, Gour R. An IoT framework for healthcare monitoring systems. Int J Comput Sci Inf Secur. IJCSIS ISSN 1947-5500. 2016;14(5), 6.

11. Krishnan DSR, Gupta SC, Choudhury T. An IoT based patient health monitoring system. 2018 International Conference on Advances in Computing and Communication Engineering (ICACCE), Paris, 2018; 01-07. https://doi.org/10.1109/ICACC E.2018.8441708.

12. Verma P, Mishra R. IoT based smart remote health monitoring system. 2020 International Conference on Electrical and Electronics Engineering (ICE3), Gorakhpur, India, 2020; pp. 467-470. https://doi.org/10.1109/ICE348803.2020.9122864.

13. Pandey H, Prabha S. Smart health monitoring system using IOT and machine learning techniques. In: 2020 Sixth International Conference on Bio Signals, Images, and Instrumentation (ICBSII), Chennai, India, 2020; pp. 1-4. https://doi.org/10.1109/ICBSI I49132.2020.9167660.

14. Banka Shubham, Madan Isha, Saranya SS. Smart healthcare monitoring using IoT. In: International Journal of Applied Engineering Research ISSN 0973-4562, 2018;13(15), 11984-11989.

15. Budida DAM, Mangrulka RS. Design and implementation of smart HealthCare system using IoT. In: 2017 International Conference on Innovations in Information, Embedded and Communication Systems (ICIIECS), Coimbatore, 2017;1-7. https://doi. org/10.1109/ICIIECS.2017.8275903.

16. Ahmed N, et al. A survey of COVID-19 contact tracing. Apps IEEE Access. 2020;8:134577-601. https://doi.org/10.1109/ ACCESS.2020.3010226.

17. Shubina V, Ometov A, Simona Lohan E. "Technical Perspectives of Contact-Tracing Applications on Wearables for COVID19 Control," 12th International Congress on Ultra Modern Telecommunications and Control Systems and Workshops (ICUMT). Brno, Czech Republic. 2020;2020:229-35. https://doi. org/10.1109/ICUMT51630.2020.9222246.

18. Hernandez-Orallo E, Manzoni P, Calafate C, Cano J-C. Evaluating how smartphone contact tracing technology can reduce the spread of infectious diseases: the case of COVID-19. IEEE Access. 2020;1-1. https://doi.org/10.1109/ACCESS.2020.29980 42.

19. Hellewell J, Abbott S, Gimma A, Bosse N, Jarvis C, Russell T, Munday J, Kucharski A, Edmunds W, Funk S, Eggo R, Sun F, Flasche S, Quilty B, Davies N, Liu Y, Clifford S, Klepac P, Jit M, Zandvoort K. Feasibility of controlling COVID-19 outbreaks by isolation of cases and contacts. Lancet Global Health. 2020;8. https://doi.org/10.1016/S2214-109X(20)30074-7.

20. https://www.serialio.com/support/learn-rfid/. Accessed on 7th November 2020.

Publisher's Note Springer Nature remains neutral with regard to jurisdictional claims in published maps and institutional affiliations. 